\title{
HLA Association with Diabetic Retinopathy - Fact or Fancy?
}

Sir,

We entirely agree with the list of criteria which should be satisfied in studies which purport to seek an association between HLA factors and diabetic retinopathy (Letter to the Editor: Barbosa \& Ramsay, Diabetologia, 1981, p 20).

In support of their argument, Barbosa and Ramsay refer to their more recent study of 200 type 1 (insulin dependent) diabetics with proliferative retinopathy [1]. An association with HLA-B15 was found in the sub-group with an age of onset of diabetes 15-40 years.

However, there are two points that should be noted: firstly many of the subjects which were used for comparison in that study had background retinopathy and whereas it might be argued that background retinopathy could have a different aetiopathogenesis compared with proliferative retinopathy, it is well known that in type 1 diabetics background changes not infrequently precede the development of new vessels. Secondly, arbitrary division of the data (e.g. age of onset subgroups) can produce chance significant results, particularly in relation to a highly polymorphic system such as HLA. The wrong conclusion is then reached that the data provide evidence for the existence of genetic heterogeneity.

We have satisfied the proposed criteria in a study of 133 longterm type 1 diabetics with severe proliferative retinopathy and 41 age and duration matched type 1 diabetics without evidence of complications. HLA-B 8 was present in $48 \%$ of the proliferative retinopathy group and in $39 \%$ of the group without complications. HLA-B15 occurred in $19 \%$ of the former and in $29 \%$ of the latter group. Not only were these differences not significant but also no significant differences were found between the groups in respect of all the HLA-A, B and C antigens tested and their GLO, Bf and C2 phenotype frequencies [2].

A combined analysis of all the data from the literature where information is quoted in relation to HLA-B8 and -B15, gives an overall frequency for all types of microvascular disease of $48 \%$ (394/824) for HLA-B8 and 29\% (213/736) for HLA-B15. Considering proliferative retinopathy alone, the available information produces an overall frequency of $47 \%(204 / 432)$ for HLA-B8 and of $28 \%(119 / 422)$ for $-B 15$. None of these frequencies differs significantly from those expected in type 1 diabetes in Caucasoids.
Thus, in attempting to study the HLA system in diabetic complications, in effect we are investigating the most polymorphic genetic system known in relation to different forms of tissue damage, whose aetiology remains obscure and almost certainly involves multiple factors. Therefore, we strongly support the concept that stringent criteria must be obeyed and that studies must be done on large numbers of well characterised patients. In addition, it needs to be emphasised that it is very difficult to demonstrate a significant additional increase of any HLA specificity relating to a secondary aspect of the disease, when that particular antigen is already known to be associated with the primary susceptibility to develop the disease, in this case type 1 diabetes.

Thus, we are compelled to conclude that at this present time there is no convincing evidence to invoke an HLA influence in the susceptibility to the microvascular complications of diabetes.

H. J. Bodansky and Eva Wolf

\section{References}

1. Barbosa J, Ramsay RC, Knobloch WH, Cantril HL, Noreen $\mathrm{H}$, King R, Yunis E (1980) Histocompatibility antigen frequencies in diabetic retinopathy. Am J Opthalmol 90:148-153

2. Cudworth AG, Bodansky $\mathrm{HJ}$ (in press) Genetic and immunological factors in diabetic complications. In: Keen $\mathrm{H}$, Jarrett $\mathbf{J}$ (eds) Complications of diabetes, 2nd edn. Edward Arnold, London

Dr. H. J. Bodansky

Department of Diabetes, Medical Unit

King George V Building (5th Floor)

St. Bartholomew's Hospital

London EC1A 7BE, UK 